POS $\quad \begin{aligned} & \text { PROCEEDINGS } \\ & \text { OF SCIENCE }\end{aligned}$

\title{
Maria Krawczyk memorial
}

\section{Jan Kalinowski}

Faculty of Physics, University of Warsaw, ul. Pasteura 5, 02-093 Warsaw, Poland

E-mail: Gan.kalinowskidfuw.edu.p]

Corfu Summer Institute 2017 'School and Workshops on Elementary Particle Physics and Gravity' 2-28 September 2017

Corfu, Greece 
Professor Maria Krawczyk passed away suddenly on 24 May 2017 in the middle of the PLANCK2017 conference. It was a shock not only for her family but also for many physicists and friends in the Faculty of Physics at the University of Warsaw and abroad. She was a very wellknown and respected scientist within the physics community for her passion and involvement in research, teaching and outreach. She was also a great enthusiast of Corfu schools and workshops, frequently attending them, giving lectures and short contributions, engaging in hot discussions and contributing to lively atmosphere.

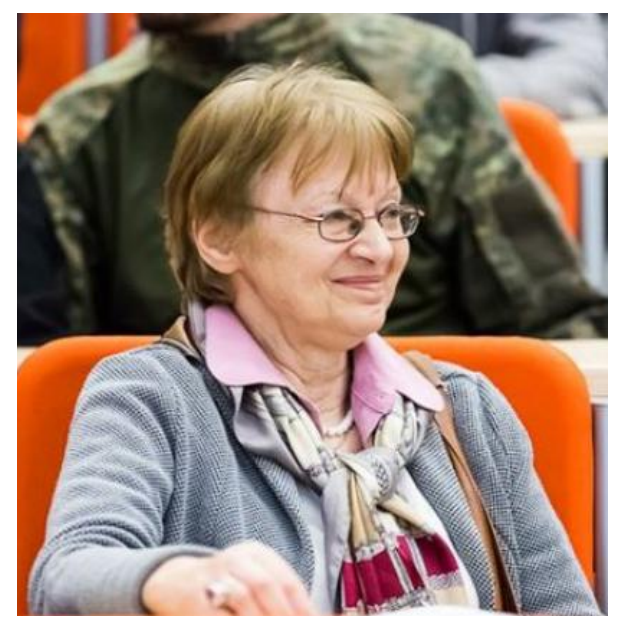

Maria graduated from the University of Warsaw in 1969. Her entire scientific career was involved with the University of Warsaw, first as an assistant, then adjunct, university professor and full professor. At the beginning of her career she worked on multihadron production processes within Regge theory. In 1975 she defended her PhD thesis under the supervision of prof. Grzegorz Białkowski based on studies of charge exchange $\pi^{-} p \rightarrow \pi^{0} n$ reaction. During a postdoc at the Max Planck Institute in Munich in 1977/78 her scientific interest shifted towards the parton model and quantum chromodynamics (QCD). She worked on photon pair production in hadron collisions and on the hadronic properties of photons within QCD. Her speculations on direct photon pair production in hard collisions were later verified by experiments. Later she worked on the resummation of higher order QCD corrections, small-x physics, structure of the photon and deep-inelastic Compton scattering.

In 1990 Maria became interested in electroweak interactions, in particular the Brout-EnglertHiggs mechanism of spontaneous electroweak symmetry breaking and the Higgs sector. The Higgs particle became her main research direction, including the two-Higgs-doublet models, searches for light Higgs particles in existing and planned accelerators, CP properties of the scalar sector, the role of the Higgs in astrophysics and cosmology, and the structure of the vacuum. She was an enthusiast for studying photon collisions at a future linear collider, and took an active role in workshops devoted to the physics potential of future experiments. During a stay at CERN in 2002 she initiated discussions and studies of CP violation in non-standard Higgs models, becoming an organiser of the workshop on CP studies and non-standard Higgs physics - which culminated in the delivery of a CERN Yellow Report. With the advent of the LHC, she concentrated mainly on the LHC physics. 
During her scientific career Maria collaborated with many distinguished physicists around the world and coordinated a number of scientific grants financed by Polish and European agencies, right up to her last project, HARMONIA. Maria served in a number of advisory committees and was involved in several international workshops and conferences. She served on the TESLA collaboration board, represented Poland in outreach within the European linear collider steering group and in 2004 was invited to join the program committee of the Rencontre de Moriond series of conferences on QCD.

Maria enjoyed teaching, liked contacts with students. She was concerned not only with their scientific developments but also with their living conditions, helped in sending them to physics schools and conferences, finding grant opportunities and helping in editing grant applications. She was very active in daily matters at the Institute and at the University and engaged heavily in outreach activities, giving interviews in radio and TV, lecturing at scientific festivals, and organising LHC exhibitions.

I knew Maria for more than 45 years. She was my best friend and collaborator. We worked together, discussed physics, attended conferences, applied for grants, organised meetings. Many times we argued, but always in a friendly atmosphere. There were no "impossible things" - she always was saying that if something is worth doing, there must be a way to achieve this. She was a great enthusiast of all technical innovations and gadgets.

Maria was a very kind and helpful person. Her advice, also in private matters, helped me to see things in a different perspective. Her kindness and friendliness will be greatly missed. She was also a beloved wife, a mother of two children and grandmother of four grandchildren. 